\title{
VIOLENCIA DE PAREJA EN HOMBRES GAY Y MUJERES LESBIANAS CHILENAS: UN ESTUDIO EXPLORATORIO*
}

\author{
INTIMATE PARTNER VIOLENCE IN CHILEAN GAY MEN AND LESBIAN WOMEN: \\ AN EXPLORATORY STUDY
}

\section{Fabiola Gómez Ojeda**, Jaime Barrientos Delgado***, Mónica Guzmán González ${ }^{* * *}$, Manuel Cárdenas Castro**** Y Joaquín Bahamondes CorRea ${ }^{* * * * *}$}

\author{
*Este trabajo forma parte del proyecto Más allá de la homofobia: Calidad de vida y crecimiento postraumático como \\ respuesta al estrés minoritario de gay y lesbianas en Chile (FONDECYT Regular $\mathrm{N}^{\circ} 1140887$ ) financiado por el Fondo \\ Nacional de Investigación Científica y Tecnológica de Chile, dirigido por Jaime Barrientos Delgado, PhD \\ y por una beca para estudios de doctorado del CONICYT otorgada a la primera autora. \\ ${ }^{* *}$ Magíster en Psicología Social y Doctoranda en Psicología. Escuela de Psicología, Pontificia Universidad Católica \\ de Chile. Santiago, Chile. E-Mail: fagomez3@uc.cl \\ ${ }^{* * *} \mathrm{PhD}$ en Psicología Social. Profesor Asociado en la Escuela de Psicología de la Universidad de Santiago. \\ Santiago, Chile. E-Mail: jaime.barrientos@usach.cl \\ ${ }^{* * * * P h D}$ en Psicología. Profesora Asociada e Investigadora en la Escuela de Psicología de la Universidad Católica \\ del Norte. Antofagasta, Chile. E-Mail: moguzman@ucn.cl \\ ${ }^{* * * *} \mathrm{PhD}$ en Psicología Social. Profesor Asociado e investigador en la Escuela de Psicología de la Universidad de \\ Valparaíso. Valparaíso, Chile. E-Mail: manuel.cardenas@uv.cl \\ ${ }^{* * * * * *}$ Maestrando en Psicología Social en la Escuela de Psicología de la Universidad Católica del Norte. \\ Antofagasta, Chile. E-Mail: joaquin.bahamondes@gmail.com
}

\section{REsUmEN}

La violencia en las relaciones de pareja del mismo sexo es un tema poco explorado. Históricamente, esta problemática ha sido estudiada en el marco de relaciones heterosexuales ubicando a las mujeres como víctimas y a los hombres como principales agresores. El propósito de este estudio fue describir las experiencias de violencia de pareja (VP) en una muestra de 268 hombres gay y 199 mujeres lesbianas. Se utilizó un muestreo tipo bola de nieve para reclutar la muestra en cuatro ciudades chilenas (Antofagasta, Valparaíso, Santiago y Concepción). Para la recolección de datos se aplicó un cuestionario especialmente diseñado para este estudio. Se realizaron análisis descriptivos y Chi-cuadrado para indagar posibles relaciones por sexo y características sociodemográficas. Entre los principales resultados se puede señalar que del total de la muestra $(N=467)$ sólo 80 personas $(17.25 \%)$ reportaron haber sido receptoras de algún tipo de VP. Se encontraron diferencias estadísticamente significativas en el reporte de violencia psicológica entre hombres gay y mujeres lesbianas $\left[\chi^{2}(1)=6.37, p=.01, \omega=.64\right]$. Los hombres gay reportaron mayor frecuencia de violencia psicológica (87.5\%) en comparación con las mujeres lesbianas (65.8\%). También se encontró una asociación estadísticamente significativa entre experiencias de VP y nivel educativo $\left[\chi^{2}(3)=10.53, p=.01, \omega=.51\right]$. Las personas con mayor nivel educativo reportan frecuencias menores de VP. Finalmente, se encontró una relación estadísticamente significativa entre haber sido víctima de VP y haber sido perpetra- 
dor de VP. Los resultados son discutidos y se plantean implicaciones para futuras investigaciones.

Palabras clave: Violencia de pareja; Relaciones del mismo sexo; Hombres gay; Mujeres lesbianas; Victimización.

\section{ABSTRACT}

The recognition of same-sex relationships has increased, but same-sex intimate partner violence has been less studied. Historically, this problem had been studied in heterosexual relationships, women being victims and men main aggressors. This heteronormative approach to intimate partner violence (IPV) often neglects same-sex relationships (Finneran, Chard, Sineath, Sullivan, \& Stephenson, 2012; Russell, 2015). For this study, IPV is defined as every act causing psychological, physical or sexual damage, within the context of intimate relationships (Harvey et al., 2007) perceived as such.

To our knowledge, there are no studies about IPV in same-sex relationships in Chile. Data about this topic is scarce in the Latin American context (Ferreira et al., 2015). Therefore, it is necessary to provide contextualized knowledge about IPV in same-sex relationships to face this psychosocial problem.

The aim of this study was to describe IPV in same-sex relationships in gay men and lesbian women and its sociodemographic characteristics. In addition, results are compared to detect possible differences between groups. LGBT populations are considered a difficult-to-reach or hidden population (Paz-Bailey et al., 2013). For this reason, a snowball sampling procedure was used. The sample consists of 467 participants who identify themselves as gay men $(57.4 \%)$ or lesbian women (42.6\%), aging from 18 to 67 years $(M=27.9$; $S D=7.9)$. The sample was recruited in four Chilean cities (Antofagasta, Valparaíso, Santiago, and Concepción). A specially designed questionnaire was administered to collect data for this study. Results were obtained through descriptive and comparative analyses with a Chi-square Test. Analyses indicate that 80 subjects $(17.2 \%)$ had experienced some form of IPV (psychological, physical, or sexual); $87.7 \%$ of them reported psychological violence; and about half of them (47.5\%) physical violence. Likewise, $19.3 \%$ respondents reported that there had been an IPV perpetrator. For group analysis, $20.1 \%$ of lesbian women and $19.3 \%$ of gay men reported some IPV experience. Statistically significant differences between gay men and lesbian women respondents were detected for psychology violence perpetrated $\left[\chi^{2}(1)=6.37, p=.01, \omega=.64\right]$. Gay men reported a higher percentage perpetrating psychological violence in their relationship (87.5\%), as compared to the group of lesbian women (65.8\%). Also, a statistically significant relation was found between IPV experiences and educational levels $\left[\chi^{2}\right.$ $(3)=10.53, p=.01, \omega=.51]$. Subjects with higher educational levels report less IPV frequency. Finally, a statistically significant relation was found between IPV victims and IPV perpetrators.

This study represents the first approach to describe and characterize IPV in gay men and lesbian women, thus creating a baseline for making comparisons with future findings on LGBT issues in Chile and Latin America. Results support evidence concerning greater prevalence of psychological violence in both gay men and lesbian women, as compared to other types of violence such as physical and sexual (Barrett \& St. Pierre, 2013; Finneran y Stephenson, 2013; Hellemans et al., 2015; Messinger, 2011). In addition, this result is similar to those of previous studies on general population (Russell, 2015).

An important result is IPV mutuality in the sample, namely, IPV occurs in two directions: persons experiencing partner violence concurrently perpetrate violence against their partners. Findings support the view that, in general, violence in samesex relationships takes place gradually from relational dynamics marked by violence (Barrientos, Rodríguez-Caballería, Escartín \& Longares, in press).

In this context, although data from this study are exploratory-descriptive, they make up a good approach to the problem since they include gay men and lesbian women from different Chilean areas of varied sociodemographic characteristics. If similar findings are reported in other studies, they could help direct psychosocial interventions, public policies, and future research. In any case, results must be carefully considered since they are non- 
representative samples and, in theory, not comparable with each other. Limitations and implications for future IPV research in same-sex relationships are discussed.

Key words: Intimate partner violence; Same-sex relationship; Lesbian; Gay men; Victimization.

\section{INTRODUCCIÓN}

El desarrollo de marcos normativos que reconocen los Derechos Humanos como principios rectores de las sociedades han promovido el interés por el estudio de la violencia en las relaciones de pareja (Harvey, Garcia-Moreno \& Butchart, 2007). La prevalencia de esta problemática lo ha posicionado como un tema de salud pública (Garcia-Moreno et al., 2006; Harvey, Garcia-Moreno \& Butchart, 2007). Sin embargo, históricamente el estudio de este tema ha sido abordado desde una perspectiva heteronormativa en el contexto de la violencia de género o violencia familiar, enfocándose en la victimización de la mujer en parejas heterosexuales (Hellemans, Loeys, Buysse, Dewaele \& de Smet, 2015; Letellier, 1994). Esto se ha traducido en la invisibilización de este tema en parejas del mismo sexo (Finneran, Chard, Sineath, Sullivan \& Stephenson, 2012; Russell 2015).

En las últimas décadas se ha observado un creciente interés por el estudio de la violencia en las relaciones de pareja entre personas del mismo sexo (Baker, Buick, Kim, Moniz \& Nava, 2013; Messinger, 2011). Sin embargo, según autores como Burke y Follingstad (1999), aún persisten percepciones culturales que conceptualizan la violencia en pareja limitada exclusivamente a un perpetrador hombre que abusa de una víctima mujer así como aún predomina el estigma hacia la población lesbiana, gay, bisexual y transgénero (LGBT) y a las relaciones íntimas entre personas del mismo sexo. Otros autores como Poorman, Seelau, E.P. y Seelau, S.M. (2003) indican que la violencia perpetrada por los hombres hacia las mujeres ha sido cataloga- da como más importante y grave que otros tipos de violencia. Dicha afirmación se sustenta en postulados del feminismo que otorgan al patriarcado las causas de la violencia masculina hacia las mujeres en las relaciones íntimas (McClennen, 2005).

En este sentido, los esfuerzos de los investigadores principalmente han estado puestos en comparar la prevalencia de este tipo de violencia en relaciones de pareja heterosexuales y entre personas del mismo sexo (Barrientos, Rodríguez-Caballería, Escartín \& Longares, en prensa; Ferreira, Simões, do Carmo \& Moura, 2015), y si bien se ha podido establecer que en ambos grupos este tipo de victimización se da con patrones similares, poco se ha avanzado en la comprensión de posibles elementos diferenciadores de la violencia de pareja en relaciones entre personas del mismo sexo (Ard \& Makadon, 2011; Balsam, Rothblum \& Beauchaine, 2005; Hellemans et al., 2015). La identificación de este tipo de elementos tiene gran importancia para el estudio y abordaje clínico de este fenómeno.

Al respecto, Ard y Makadon (2011) han señalado que la no develación de la orientación sexual por temor a ser discriminado, puede ser en sí misma una herramienta para la victimización, al mismo tiempo que se traduce en una barrera para pedir apoyo. También han señalado que experiencias previas de victimización en otros contextos (escuela, familia, trabajo, etc.) pueden favorecer una menor sensibilidad frente a este tipo de violencia. Por tanto, hacer investigaciones sobre la violencia en parejas del mismo sexo supone ir más allá del paradigma heterosexual que ha sustentado generalmente los estudios en este tema (Ristock, 2003).

Por otra parte, en Chile y Latinoamérica tampoco se tiene conocimiento de estudios que aborden el tema en parejas del mismo sexo y la información existente en el contexto latinoamericano es escasa (Ferreira et al., 2015), por lo que resulta urgente aportar conocimiento contextualizado que permita afrontar este problema psicosocial.

Chile aprobó en el año 2012 una ley antidiscriminación y en el año 2015 una ley que 
regula las uniones civiles entre parejas del mismo sexo. Además, en el país, según un reciente estudio hecho en el marco de la marcha del orgullo LGBT del 2011, un 59.4\% de las personas encuestadas estaba en algún tipo de relación de pareja (Barrientos \& Bozón, 2014). Por tanto, habría una proporción importante de la población LGBT que podría estar afectada por este fenómeno, lo que releva la importancia de su estudio.

\section{VIOLENCIA DE PAREJA EN RELACIONES DE PERSONAS DEL MISMO SEXO}

Se ha definido a la violencia de pareja (en inglés intimate partner violence - IPV) como cualquier comportamiento dado dentro de una relación de intimidad afectiva que causa daño psicológico, físico o sexual a los miembros de la relación (Harvey et al., 2007). Sin embargo, no hay un consenso en su definición y operacionalización (cf., Barrientos et al., en prensa), lo que se ha traducido en hallazgos variados con respecto a su prevalencia, características y consecuencias de este problema psicosocial (cf., Finneran \& Stephenson, 2014; Hellemans et al., 2015; Messinger, 2011; Owen \& Burke, 2004).

Investigaciones recientes, como la efectuada por Barrett y St. Pierre (2013), con una muestra de personas LGB (lesbianas, gay y bisexuales) canadienses, reporta que uno de cada tres personas es víctima de violencia financiera o emocional y que una decada cinco ha experimentado violencia física o sexual. En tanto, un estudio realizado con parejas de mujeres reporta un $11 \%$ de violencia en la pareja (Messinger, 2011). Hellemans y colaboradores (2015) en una muestra de hombres y mujeres con pareja del mismo sexo encontraron que el $14.5 \%$ de las personas encuestadas reportó agresión física y más de la mitad, violencia psicológica en su actual pareja $(57.9 \%)$.

Estas diferencias en la proporción de violencia reportada, no solo tienen relación con la variedad de definiciones con las que se aborda la violencia en la relación de pareja (VP), sino también con diferencias metodo- lógicas como las muestras y los instrumentos de medición utilizados, entre otros aspectos necesarios de considerar (Finneran \& Stephenson, 2013; Turell, 2000).

Sin embargo, se evidencia cierto consenso en señalar que el tipo de VP reportado con mayor frecuencia en parejas del mismo sexo es la violencia psicológica (Barrett \& St. Pierre, 2013; Burke, Jordan \& Owen, 2002; Finneran \& Stephenson, 2013; Hellemans et al., 2015; Messinger, 2011; Owen \& Burke, 2004; Turell, 2000).

Por otro lado, las diferencias en la prevalencia de VP entre hombres gay y mujeres lesbianas ha sido poco explorada (Matte \& Lafontaine, 2011). Algunos estudios han reportado una mayor prevalencia de VP entre hombres gay en comparación con mujeres lesbianas (ver Burke et al., 2002), otros reportan una mayor frecuencia de victimización en parejas lesbianas en comparación con hombres gay (Barrett \& St. Pierre, 2013; Hellemans et al., 2015; Turell, 2000). Existen otros estudios que no encuentran diferencias entre estos dos grupos (Messinger, 2011; Owen \& Burke, 2004). Dado que no existe consenso sobre el tema, algunos autores proponen avanzar en el estudio diferencial de dinámicas de violencia en parejas mujer-mujer y hombre-hombre (Cannon \& Buttell, 2015).

Por otro lado, se ha visto que la perpetración de VP en minorías sexuales ha sido poco explorada (Barrientos et al., en prensa; Finneran \& Stephenson, 2013). Al respecto, un estudio realizado en seis países reporta rangos de violencia física perpetrada que va entre $2.47 \%$ y $5.76 \%$ en parejas del mismo sexo (Finneran et al., 2012). Por su parte, Edwards y Sylaska (2013) señalan que más de la mitad de los encuestados en su estudio no reporta violencia alguna, el $13.3 \%$ reporta haber sido victimizado y sólo el 7.3\% haber ejercido violencia de pareja. FRECUENCIA REPORTADA DE VIOLENCIA DE PAREJA Y
CARACTERÍSTICAS SOCIODEMOGRÁFICAS

Cuando la investigación es incipiente con respecto a un fenómeno, un primer nivel de 
análisis es el descriptivo, de allí el énfasis en el relevamiento de variables sociodemográficas para el estudio de este tipo de violencia. Este sería el estado actual de la investigación de este fenómeno, en particular en América Latina (Barrientos et al., en prensa).

Se ha vinculado la violencia de pareja en relaciones de personas del mismo sexo con algunas características sociodemográficas. Sin embargo, el estudio realizado por Finneran y colaboradores (2012) en seis países (Estados Unidos, Australia, Canadá, Sudáfrica, Reino Unido y Brasil) no obtuvo resultados consistentes en las distintas muestras. Por ejemplo, los autores señalan que la VP se relaciona significativamente con la edad en Sudáfrica (a menor edad, mayor prevalencia de VP) y con el nivel educativo en Australia (a menor nivel educativo, mayor prevalencia de VP), pero no en los otros países incluidos en el estudio. Por su parte, Barrett y St. Pierre (2013) reportan que las personas que tienen menor edad y menor nivel educativo han experimentado mayor frecuencia de VP. Otros estudios no han encontrado diferencias estadísticamente significativas en función de la edad de los encuestados.

Hellemans y colaboradores (2015) señalan que el reporte de violencia física en parejas del mismo sexo disminuye ante el mayor nivel educacional, y que tanto las experiencias de violencia psicológica como las de violencia sexual se incrementan con la edad y disminuyen con un mayor nivel educacional.

Los resultados descritos permiten comprender el desafío que implica el estudio de la VP en parejas del mismo sexo y la necesidad de explorar este campo con mayor profundidad.

De este modo, se realizó un estudio de carácter exploratorio-descriptivo, que tuvo como objetivo describir la violencia de pareja en hombres gay y mujeres lesbianas chilenas y explorar las posibles diferencias entre ambos grupos, como así también las características sociodemográficas asociadas al fenómeno. Para tales efectos, se ha definido a la violencia de pareja como cualquier comportamiento dado dentro de una relación de intimidad afectiva que causa daño psicológico, físico o sexual a los miembros de la relación (Harvey et al., 2007) y que es percibida como tal.

\section{MÉTOdO}

\section{PARTICIPANTES}

Este estudio contó con una muestra de 467 sujetos que se autoidentificaron como hombres gay y mujeres lesbianas, cuyas edades fluctuaban entre 18 y 67 años $(M=27.9$; $D E=7.9)$. La muestra quedó constituida por 268 hombres gay $(57.4 \%)$, con edades entre 18 y 67 años $(M=27.6 ; D E=7.6)$ y 199 mujeres lesbianas $(42.6 \%)$, con edades entre 18 y 60 años $(M=28.1, D E=8.2)$.

Las minorías sexuales son consideradas como muestras de difícil acceso (Paz-Bailey et al., 2013), por tanto, no se cuenta con marcos muestrales que permitan seleccionar muestras aleatorias. Por esta razón, la muestra fue seleccionada a partir de un muestreo en cadena tipo bola de nieve, tal como se ha realizado en estudios previos en la región (Barrientos \& Cárdenas, 2014; Barrientos, Cárdenas \& Gómez, 2014).

Los criterios de selección fueron la autoidentificación sexual (hombres gay y mujeres lesbianas) y la ciudad de residencia. En cuanto a la ciudad de residencia, se recolectaron datos de cuatro ciudades chilenas (Antofagasta, Valparaíso, Santiago y Concepción) consideradas representativas de las distintas zonas de Chile (centro, norte y sur) y donde las organizaciones LGTB (lésbicas, gay, transgénero y bisexuales) nos facilitaron el acceso a la población objeto de estudio.

\section{INSTRUMENTOS}

La mayoría de las variables usadas han sido pilotadas en estudios previos (ONUSIDA, Organización de las Naciones Unidas para la Educación, la Ciencia y la Cultura, Chile, Ministerio de Salud, VIVOPOSITIVO y ASOSIDA, 2012). En particular, las medidas referidas a VP fueron tomadas de estudios 
previos realizados sobre el tema, pero en población heterosexual (Guzmán, Espinoza, Tay, Leiva \& Adaos, 2014).

Se preguntó a los encuestados por su autoidentificación sexual (¿Cómo te identificas sexualmente?), edad en años cumplidos al momento de la entrevista (esta variable fue re-categorizada en dos grupos: 18-29 años y 30 años o más), ciudad de residencia (Antofagasta, Santiago, Valparaíso o Concepción) y nivel educacional (¿Cuál es el nivel educativo más alto que has alcanzado?, con opciones de respuesta que van desde educación básica incompleta a educación de postgrado); esta variable fue recategorizada en 4 grupos: educación básica y educación media incompleta (EBC-EMI), educación media completa y educación universitaria incompleta (EMC-EUI), educación universitaria completa y educación de post-grado, e identificación religiosa ( $₫$ Te consideras una persona religiosa?, con opción de respuesta dicotómica: sí / no).

Además, se midió el nivel socioeconómico (NSE) a partir del indicador ESOMAR (European Society for Opinion and Marketing Research), medida adaptada para el contexto chileno por ADIMARK (2014). Esta medida evalúa el nivel económico y social de las familias en comparación con otras a partir de una matriz de seis niveles que combina el nivel educativo y la categoría ocupacional del principal sostenedor del hogar. Para facilitar el análisis de los datos esta variable fue recategorizada en tres niveles: NSE bajo, NSE medio y NSE alto.

\section{RELACIONES DE PAREJA}

Se consultó a los encuestados sobre su estatus de pareja (¿En la actualidad tienes una pareja de tu mismo sexo?, con opciones de respuesta sí / no), sobre el tiempo de duración de la relación en meses (¿Cuánto tiempo de relación tienen?), sobre cohabitación (¿En la actualidad vives con tu pareja?, con opciones de respuesta sí / no) y sobre parentalidad (¿Tienes hijos?, ¿Vives con ellos? con opciones de respuesta dicotómica sí / no).

\section{VIOLENCIA DE PAREJA (VP)}

Las preguntas de este apartado fueron construidas especialmente para este estudio, en función del tipo de violencia mayormente explorada y reportada por las investigaciones revisadas. Para indagar violencia recibida se preguntó: ¿Has sido víctima de violencia psicológica, física o sexual por parte de tu pareja actual? En aquellos casos en que no estaba en pareja se preguntó por la última relación que tuvo (con formato de respuesta dicotómico: sí / no). Adicionalmente, solo a aquellos que respondieron sí a la pregunta señalada, se preguntó por el tipo de violencia recibida: psicológica, física y sexual, con opciones de respuesta dicotómica ( sí / no por cada tipo de violencia).

Del mismo modo, para indagar acerca de la violencia ejercida se preguntó: ¿Has actuado alguna vez de modo violento contra tu pareja actual, ya sea que se trate de violencia psicológica, física o sexual?

En los casos en que no estaba en pareja se preguntó por la última relación (el formato de respuesta fue dicotómico: sí / no). Adicionalmente, solo a aquellos que respondieron sí a la pregunta señalada, se les preguntó el tipo de violencia ejercida: psicológica, física y sexual, con opciones de respuesta dicotómica (sí / no por cada tipo de violencia).

\section{Procedimiento}

Este estudio es el primero que aborda la VP en minorías sexuales en el país y forma parte de un proyecto mayor que busca medir el estigma y discriminación hacia hombres gay y mujeres lesbianas en Chile y sus efectos en la calidad de vida y el bienestar. Por lo tanto, se utilizó un cuestionario con diversas medidas, muchas de las cuales no fueron utilizadas para este estudio ${ }^{1}$.

1 El listado completo puede ser solicitado al segundo autor. 
El estudio fue realizado por un grupo de psicólogos clínicos y sociales, quienes seleccionaron un coordinador de terreno y al menos cinco encuestadores, hombres y mujeres estudiantes de Psicología y Sociología (entre los encuestadores se contó con personas gay, lesbianas y heterosexuales). Los encuestadores y el coordinador en terreno fueron capacitados por el equipo de investigación tanto para la aplicación de la encuesta como para evaluar la necesidad de derivación a apoyo psicosocial.

La administración fue individual, con una duración de 45 minutos aproximadamente. Antes de ser encuestados, a los participantes se les informaron los objetivos del estudio y firmaron una carta de consentimiento informado, donde aceptaban participar en el estudio y se les aseguraba confidencialidad y anonimato.

Para la selección de la muestra se definió un perfil para las semillas de entrevistados iniciales y a partir de ellas se comenzó la recolección de datos. Los criterios de selección para estas semillas fueron: (a) la autoidentificación como hombre gay o mujer lesbiana, (b) tener una amplia red social (personas que declaren conocer a un amplio número de personas gay o lesbianas), (c) residir en Antofagasta, Valparaíso, Santiago o Concepción y (d) ser mayor de 18 años. No se incluyeron personas bisexuales pues no estaba considerado entre los objetivos del estudio mayor. Además, se ha reportado una baja prevalencia de este grupo en Chile (Barrientos et al., 2008; Barrientos, Silva, Catalán, Gómez \& Longueira, 2010).

En cada ciudad, la recolección de la muestra comenzó con 3 semillas de hombres gay y 3 de mujeres lesbianas, excepto en Santiago (ciudad capital) donde se comenzó con 6 semillas de hombres gay y 6 de mujeres lesbianas. En todos los casos, las semillas fueron seleccionadas a partir de informantes clave. Al finalizar cada entrevista se les solicitó que invitaran a participar del estudio a 3 personas que cumplieran con los criterios de inclusión. Si las nuevas semillas accedían a participar se los contactaba, se explicaban los alcances del estudio y se repetía el procedimiento, esto hasta completar la recolección de datos en cada una de las ciudades.

Este estudio fue aceptado por la Comisión de Ética de la Universidad Católica del Norte y el Fondo Nacional de Desarrollo Científico y Tecnológico de Chile (FONDECYT). Según los procedimientos éticos, en las cartas de consentimiento informado entregadas a los participantes se indicaban lugares de consulta y derivación para las personas que demandasen más información sobre el tema o ayuda profesional específica. Asimismo, los encuestadores fueron entrenados para apoyar y orientar en caso necesario a los encuestados.

\section{Resultados}

Los datos fueron analizados según el siguiente orden: en primer lugar se realizaron análisis de frecuencia para cada variable incluida en el estudio, tanto para la muestra total como para cada grupo (hombres gay y mujeres lesbianas). Posteriormente y a fin de analizar la relación entre variables categóricas, se realizaron análisis de Chi cuadrado y se calculó el tamaño del efecto para estos análisis. Para los análisis se utilizaron el software estadístico SPSS, versión 20.0 y G*Power 3.1 .

\section{CARACTERÍSTICAS SOCIODEMOGRÁFICAS}

Las características sociodemográficas de los encuestados se resumen en la Tabla 1. En ella se presentan los resultados tanto para el total de la muestra como para hombres gay y mujeres lesbianas.

Como puede observarse en la Tabla 1, se trata de una muestra con mayor porcentaje de gente joven, en su mayoría residentes en Santiago (capital del país) y con 12 años de estudios o más $(96 \%)$, mientras que la mayor parte de los participantes (87.8\%) se sitúa entre nivel socioeconómico medio y alto.

Si se observan los resultados obtenidos para cada grupo (hombres gay y mujeres lesbianas), se puede señalar que los hombres gay reportan mayor nivel educacional ya que 
cerca del $40 \%$ ha alcanzado estudios universitarios completos y estudios de postgrado, mientras que el $33.7 \%$ de las mujeres lesbianas alcanzó estudios universitarios o superiores. Por otro lado, el $33.2 \%$ de las personas gay y un $26.7 \%$ de personas lesbianas reportaron NSE alto.

En cuanto a las relaciones de pareja, más de la mitad de los encuestados $(55.5 \%)$ se encuentra en una relación con una persona de su mismo sexo, con casi 3 años de tiempo medio de duración $(M=32.15$ meses, $D E=41.21)$. De ellos, aproximadamente 1 de cada 4 personas vive con su pareja en la actualidad.

Si se analizan los datos por grupo, 135 mujeres lesbianas $(67.8 \%)$ declaran tener una relación con una persona de su mismo sexo y un $30.7 \%$ de ellas reporta vivir con esta pareja (tiempo de duración en meses: $M=30.23, D E=29.07)$. En tanto, 124 hombres gay reportan tener una pareja del mismo sexo $(46.3 \%)$ y sólo el $18.3 \%$ reporta vivir con esta pareja (tiempo de duración en meses: $M=30.23, D E=29.07)$.

Solo 25 personas del total de los encuestados tienen hijos (5.4\%) y en su mayoría se trata de mujeres lesbianas. De aquellos que reportan tener hijos, el 77.8\% vivía con ellos al momento de ser encuestados.

\section{VIOLENCIA DE PAREJA}

Tal como se reporta en la Tabla 2, el $17.2 \%$ de los encuestados señala haber sido víctima de algún tipo de violencia (psicológica, física o sexual) en su relación de pareja actual o en su última relación (en caso de no estar en pareja). Al realizar el análisis diferenciado por autoidentificación sexual, se constata que el grupo de mujeres lesbianas reporta una mayor frecuencia de victimización en su relación de pareja $(20.1 \%)$ en comparación con los hombres gay. Sin embargo, esta diferencia no es estadísticamente significativa $\left[\chi^{2}(1)=1.01, p=.32\right]$.

En cuanto al tipo de violencia reportado, la mayor parte de ellas reporta violencia psicológica $(87.7 \%)$, seguida de violencia física $(47.5 \%)$. En el reporte por sexo, el $90 \%$ de los hombres gay (sobre un total de 40) ex- presó haber sido receptor de violencia psicológica y el 55.3\% reporta violencia física. El porcentaje de hombres gay que informa haber sido víctima de violencia sexual $(13.2 \%)$ es superior a lo reportado por la muestra total $(7.5 \%)$.

Por otro lado, el $19.3 \%$ del total de los encuestados informa haber perpetrado violencia en su relación de pareja actual o en la última que tuvo (en caso de no estar en pareja en la actualidad) (ver Tabla 2). Con mayor frecuencia se reporta haber perpetrado violencia psicológica $(78.7 \%)$, tanto a nivel general como en el análisis según sexo. Tal como puede observarse en la Tabla 2, el reporte de violencia perpetrada es muy similar entre hombres gay y mujeres lesbianas $(19.4 \%$ y $19.1 \%$, respectivamente), no encontrándose diferencias estadísticamente significativas $\left[\chi^{2}\right.$ $(1)=.18, p=.72]$. El grupo de hombres es el que reporta mayor frecuencia de violencia psicológica y física $(87.5 \%$ y $50 \%$, respectivamente). En cuanto al grupo de mujeres, llama la atención el 37.8\% de violencia física ejercida y el $2.7 \%$ de violencia sexual, esta última frecuencia es superior a la reportada por los hombres gay $(1.9 \%)$.

Con el propósito de determinar si existe alguna relación entre autoidentificación sexual y violencia de pareja se realizó un análisis de Chi cuadrado. Sólo se obtienen resultados estadísticamente significativos para violencia psicológica ejercida $\left[\chi^{2}(1)=6.37\right.$, $p=.01, \omega=.64]$. Los hombres gay reportan en un mayor porcentaje haber perpetrado violencia psicológica en su relación de pareja $(87.5 \%)$ en comparación con el grupo de mujeres lesbianas $(65.8 \%)$.

\section{VARIABLES SOCIODEMOGRÁFICAS Y VIOLENCIA DE PAREJA}

Se encuentra una relación entre haber recibido violencia de pareja y el nivel de estu$\operatorname{dios}\left[\chi^{2}(3)=10.53, p=.01, \omega=.51\right]$. Las personas que tienen mayor educación reportan menor frecuencia de victimización en la pareja. La frecuencia reportada por las personas que tienen estudios universitarios completos y estudios de postgrado es de $9.5 \%$ y $16.7 \%$, 
respectivamente. Por su parte, los grupos que tienen EBC-EMI y EMC-EUI son los que reportan con mayor frecuencia haber recibido violencia de pareja $(22.2 \%$ y $23.1 \%$, respectivamente).

También se encuentra una relación estadísticamente significativa entre haber recibido violencia de pareja y haberla perpetrado $\left[\chi^{2}(1)=101.28, p<.001, \omega=.9\right]$. Aquellas personas que declaran haberla recibido reportan con mayor frecuencia haberla perpetrado $(63.3 \%)$ en comparación con aquellas que no la han recibido $(11.7 \%)$.

Realizando los análisis segmentados por sexo, en el caso de las personas gay se encuentra una relación estadísticamente significativa entre haber recibido violencia de pareja y el nivel de estudios de los encuestados $\left[\chi^{2}(3)=9.99, p=.02, \omega=.8\right]$. Las personas que tienen EBC-EMI y EMC-EUI son las que reportan mayor frecuencia de victimización por parte de su pareja (18.2\% y $22.8 \%$, respectivamente). Por su parte, las personas que reportan una menor frecuencia son las que tienen estudios universitarios completos $(5.6 \%)$ y estudios de postgrado $(16.7 \%)$.

Por otro lado, se encontró una relación estadísticamente significativa entre haber perpetrado violencia de pareja y haberla recibido $\left[\chi^{2}(1)=36.30, p<.001, \omega=.7\right]$. Los hombres gay que declaran haberla perpetrado, reportan en un $59 \%$ haberla recibido, pero sólo el $14.9 \%$ de los que la han perpetrado, no han sido víctimas. De igual modo, para el grupo de mujeres lesbianas se encuentra una relación estadísticamente significativa entre haber perpetrado violencia de pareja y haberla recibido $\left[\left(\chi^{2}(1)=69.95, p<.001, \omega=1.3\right]\right.$. Las mujeres lesbianas que han declarado haberla perpetrado dentro de la relación de pareja, reportan en un $67.5 \%$ de los casos haberla recibido y sólo un $7.5 \%$ de las que declaran haberla perpetrado no han sido víctimas.

\section{DISCUSIÓN}

Los resultados obtenidos apoyan la evidencia existente con respecto a la mayor pre- valencia de violencia psicológica tanto en hombres gay como en mujeres lesbianas en comparación con otros tipos de violencia como la física y sexual (Barrett \& St. Pierre, 2013; Burke et al., 2002; Finneran \& Stephenson, 2013; Hellemans et al., 2015; Messinger, 2011; Owen \& Burke, 2004; Turell, 2000). Por otro lado, este resultado es similar a lo reportado por estudios previos con población general (Russell, 2015).

En cuanto a patrones diferenciados por sexo, sólo se encuentran diferencias estadísticamente significativas para la perpetración de violencia psicológica, donde los hombres gay reportan en mayor medida haber perpetrado violencia psicológica a su pareja en comparación con las mujeres lesbianas. Este hallazgo quizás se asociaría a la mayor adhesión, por parte de hombres gay con respecto a mujeres lesbianas chilenas, a modelos de socialización de género rígidos que reproducen modos violentos de resolución de conflictos en el ámbito íntimo (Barrientos, 2015) o dinámicas de poder y de control propios de modelos heteronormativos (Burke \& Follingstad, 1999). Futuros estudios deberían profundizar sobre este tema, toda vez que algunas investigaciones han propuesto que las relaciones de pareja entre personas del mismo sexo tenderían a ser más igualitarias (Schechory \& Ziv, 2007).

Por otro lado, la frecuencia reportada a nivel general (haber sido víctima de algún tipo de violencia, entre $14 \%$ y $20 \%$ ) es muy inferior a lo reportado por otros estudios (por ej., Barrett \& St. Pierre, 2013; Owen \& Burke, 2004). Esta menor prevalencia puede estar relacionada con el tipo de instrumento utilizado para este estudio para medir VP, en tanto se trata de una pregunta única y que está permeada por lo que cada persona considera violencia de pareja y sus subtipos. En futuros estudios es necesario incorporar otro tipo de instrumentos (por ejemplo, Conflict Tactic Scale) para evaluar si esta diferencia persiste.

En cuanto a la relación entre VP y variables sociodemográficas, los resultados obtenidos son congruentes con lo reportado por otros estudios que encuentran una relación 
entre bajo nivel educativo y mayor frecuencia de VP (Barrett \& St. Pierre, 2013; Hellemans et al., 2015). No obstante, no se encuentran diferencias significativas entre VP y edad de los encuestados, tal como ha sido reportado por Burke y colaboradores (2002). Esta información sociodemográfica es útil para describir la violencia de mejor modo y avanzar en su eventual prevención focalizando dicho trabajo en determinados grupos donde ella sea más prevalente. Pero también, relevar que el fenómeno se asocia a algunas variables más que a otras, permite disponer de información útil para estudios futuros que busquen explicar el fenómeno (Barrientos et al., en prensa).

Un hallazgo relevante es el fenómeno de mutualidad reportado, es decir, un porcentaje importante de quienes han sido receptores de VP también la han perpetrado. Esta bidireccionalidad sugiere que la violencia de pareja en relaciones del mismo sexo, en ciertas circunstancias, tiene lugar de manera gradual a partir de dinámicas relacionales marcadas por la violencia (Barrientos et al., en prensa). Si bien son escasos los estudios que abordan ambos tipos de violencia (recibida y perpetrada) (Ferreira et al., 2015), existen estudios que han problematizado este aspecto tratando de diferenciarlo de dinámicas de autodefensa (Coker, Smith, McKeown \& King, 2000; Morrow, 1994; Ristock, 2003). En este sentido, un estudio realizado con jóvenes chilenos revela una alta prevalencia de mutualidad en violencia de tipo psicológica, sugiriendo que podría tratarse de una estrategia de enfrentamiento del conflicto en la relación de pareja (Guzmán et al., 2014). Por su parte, un estudio realizado con parejas del mismo sexo, que identificó altos niveles de correlación entre haber perpetrado y recibido violencia psicológica, sugiere que es posible que individuos con comportamientos violentos influencien a sus parejas a comportarse violentamente (Matte \& Lafontaine, 2011). En cualquier caso, es un tema que debe ser abordado por futuras investigaciones de manera específica, indagando en posibles patrones diferenciales de VP entre parejas heterosexuales y del mismo sexo.
En Chile, sólo recientemente se ha admitido el derecho de las parejas del mismo sexo a convivir y formar una familia a partir de la aprobación de la Ley de Acuerdo de Unión Civil (promulgada en abril de 2015 y en vigencia desde octubre de 2015). La nueva normativa reconoce a las parejas del mismo sexo, que así lo soliciten, el estatus de núcleo familiar y conforme a ello, protege a la pareja en diversas materias (familiares, patrimoniales, previsionales y derechos de salud, entre otros). Sin embargo, la normativa legal que abarca a la VP refiere a leyes sobre violencia intrafamiliar (VIF), cuyo origen y fundamento es claramente heteronormativo. Los resultados de este estudio y la evidencia acumulada en otros contextos refuerzan la necesidad de contar con normativas específicas que incorporen la variabilidad de nuestra realidad social, abarcando las especificidades de todos los tipos de familias, incluidas aquellas conformadas por parejas del mismo sexo.

Hace dos décadas Letellier (1994) llamaba la atención sobre la necesidad de ir más allá de modelos heteronormativos en el estudio de la violencia de pareja en relaciones del mismo sexo. Si bien se ha avanzado en esta línea, los resultados obtenidos por las investigaciones que abordan el tema han sido discrepantes. Estas divergencias pueden tener que ver tanto con aspectos metodológicos como con las dificultades que implica trabajar con poblaciones de difícil acceso como lo es la población LGBT. En este contexto, los datos proporcionados por este estudio, si bien son de carácter exploratorio-descriptivo, son una buena aproximación al problema, en tanto incorpora a hombres gay y mujeres lesbianas de diversas zonas de Chile y con características sociodemográficas variadas. De replicarse hallazgos similares en futuros estudios, esto puede permitir la orientación de intervenciones psicosociales, políticas públicas y futuras investigaciones.

En el ámbito psicológico clínico, los datos aportados pueden permitir comprender las particularidades de la VP en relaciones de personas del mismo sexo y orientar las intervenciones en esta materia. En este sentido, es especialmente importante considerar el por- 
centaje de mutualidad reportada por los encuestados y los patrones diferenciados por sexo.

En síntesis, este estudio es uno de los pocos existentes a nivel latinoamericano que aborda el tema de la VP entre personas del mismo sexo, dado que la gran proporción de investigaciones previas sobre este fenómeno está referida a personas heterosexuales. Identificar similitudes y diferencias en los patrones de VP resulta relevante al momento de diseñar planes de prevención e intervención que sean acordes a las características particulares de la población objetivo. En tal sentido, este estudio es un primer paso en esta línea. Además, a diferencia de investigaciones previas que evaluaron la violencia a partir de una serie de conductas predefinidas como VP (por ejemplo, el CTS, instrumento ampliamente utilizado a nivel internacional), el estudio realizado toma en consideración la percepción subjetiva de haber sido víctima o perpetrador/a de VP.

Por otro lado, es necesario considerar las limitaciones de este estudio. Una de las principales es el tipo de muestreo utilizado, a partir del cual no es posible abordar los resultados como prevalencia de VP. Una de las principales dificultades de las investigaciones que trabajan con muestras de difícil acceso, como son las minorías sexuales, es acceder a muestras representativas y a tamaños mues- trales que permitan llegar a conclusiones sólidas. Esta dificultad es muy difícil de abordar en sociedades como la chilena, donde el prejuicio y la discriminación hacia las minorías sexuales es aún muy importante (Barrientos \& Bozón, 2014; Barrientos et al., 2008; Barrientos et al., 2010).

Otro elemento a considerar es la operacionalización de la VP, pues se ha señalado que los estudios en este campo son sensibles a las definiciones a partir de las cuales se aborde el tema (Barrientos et al., en prensa) y esto podría contribuir a que se obtengan resultados diversos y muchas veces, discrepantes. Por lo tanto, es necesario incorporar en futuras investigaciones preguntas que permitan precisar el tiempo en que ocurrieron los hechos y su frecuencia; al mismo tiempo, delimitar claramente entre hechos ocurridos en la actual pareja y aquellos que pueden haber ocurrido en relaciones anteriores. Del mismo modo, considerando los beneficios del autorreporte en este campo, es necesario incluir en el instrumento de medición una mayor precisión con respecto a cuáles son las conductas consideradas violentas.

Finalmente, es pertinente abordar el tema vinculando otras variables relevantes, por ejemplo estrés minoritario, prejuicio, discriminación, estigma, homofobia internalizada, así como sus efectos en bienestar y salud mental. 
TABLA 1

VARIABLES SOCIODEMOGRÁFICAS PARA LA MUESTRA TOTAL Y POR GRUPO

(HOMBRES GAY Y MUJERES LESBIANAS)

\begin{tabular}{|c|c|c|c|c|c|c|}
\hline \multirow[t]{2}{*}{ Variables } & \multicolumn{2}{|c|}{ Gay } & \multicolumn{2}{|c|}{ Lesbianas } & \multicolumn{2}{|c|}{ Total } \\
\hline & $N$ & $\%$ & $N$ & $\%$ & $N$ & $\%$ \\
\hline \multicolumn{7}{|l|}{ Edad } \\
\hline 18-29 años & 187 & 69.8 & 138 & 69.3 & 325 & 69.6 \\
\hline 30-67 años & 81 & 30.2 & 61 & 30.7 & 142 & 30.4 \\
\hline \multicolumn{7}{|l|}{ Ciudad de residencia } \\
\hline Santiago & 128 & 47.8 & 70 & 35.2 & 198 & 42.2 \\
\hline Valparaíso & 66 & 24.6 & 44 & 22.1 & 110 & 23.6 \\
\hline Concepción & 26 & 9.7 & 39 & 19.6 & 65 & 13.9 \\
\hline Antofagasta & 48 & 17.9 & 46 & 23.1 & 94 & 20.1 \\
\hline \multicolumn{7}{|l|}{ Nivel de estudios } \\
\hline EBC y EMI & 11 & 4.1 & 7 & 3.5 & 18 & 3.9 \\
\hline EMC y EUI & 151 & 56.3 & 124 & 62.3 & 275 & 58.9 \\
\hline Universitario completo & 85 & 31.8 & 55 & 27.6 & 140 & 30.0 \\
\hline Postgrado & 21 & 7.8 & 12 & 6.1 & 33 & 7.1 \\
\hline Datos perdidos & -- & -- & 1 & 0.5 & 1 & 0.2 \\
\hline \multicolumn{7}{|l|}{ Nivel socieconómico } \\
\hline NSE Bajo & 25 & 9.3 & 17 & 8.5 & 42 & 9.0 \\
\hline NSE Medio & 147 & 54.9 & 121 & 60.8 & 268 & 57.4 \\
\hline NSE Alto & 89 & 33.2 & 53 & 26.7 & 142 & 30.4 \\
\hline Datos perdidos & 7 & 2.6 & 8 & 4.0 & 15 & 3.2 \\
\hline \multicolumn{7}{|l|}{ Identificación religiosa } \\
\hline Sí & 62 & 23.1 & 49 & 24.6 & 111 & 23.8 \\
\hline No & 201 & 75.0 & 147 & 73.9 & 348 & 74.8 \\
\hline Datos perdidos & 5 & 1.9 & 3 & 1.5 & 8 & 1.7 \\
\hline
\end{tabular}

\section{Notación:}

EBC: Educación básica completa

EMI: Educación media incompleta

EMC: Educación media completa

EUI: Educación universitaria incompleta 
Violencia de pareja en hombres gay y mujeres lesbianas

TABLA 2

VIOLENCIA DE PAREJA

CARACTERIZACIÓN POR GRUPO (HOMBRES GAY Y MUJERES LESBIANAS)

\begin{tabular}{|c|c|c|c|c|c|c|}
\hline \multirow{2}{*}{ Violencia } & \multicolumn{2}{|c|}{ Gay } & \multicolumn{2}{|c|}{ Lesbianas } & \multicolumn{2}{|c|}{ Total } \\
\hline & $N$ & $\%$ & $N$ & $\%$ & $N$ & $\%$ \\
\hline \multicolumn{7}{|l|}{ Violencia recibida } \\
\hline Sí & 40 & 14.9 & 40 & 20.1 & 80 & 17.2 \\
\hline No & 197 & 73.5 & 152 & 76.4 & 349 & 74.7 \\
\hline Datos perdidos & 31 & 11.6 & 7 & 3.5 & 38 & 8.1 \\
\hline \multicolumn{7}{|c|}{ Tipo de violencia recibida ${ }^{a}$} \\
\hline Psicológica & 36 & 90.0 & 35 & 85.4 & 71 & 87.7 \\
\hline Física & 21 & 55.3 & 17 & 40.5 & 38 & 47.5 \\
\hline Sexual & 5 & 13.2 & 1 & 2.4 & 6 & 7.5 \\
\hline \multicolumn{7}{|l|}{ Violencia ejercida } \\
\hline Sí & 52 & 19.4 & 38 & 19.1 & 90 & 19.3 \\
\hline No & 184 & 68.7 & 149 & 74.9 & 333 & 71.3 \\
\hline Datos perdidos & 32 & 11.9 & 12 & 94.0 & 44 & 9.4 \\
\hline \multicolumn{7}{|c|}{ Tipo de violencia ejercidaa } \\
\hline Psicológica & 49 & 87.5 & 25 & 65.8 & 74 & 78.7 \\
\hline Física & 27 & 50 & 14 & 37.8 & 41 & 45.1 \\
\hline Sexual & 1 & 1.9 & 1 & 2.7 & 2 & 2.2 \\
\hline
\end{tabular}

aPara cada tipo de violencia se reportan frecuencias y porcentajes referidos a los que han declarado haber recibido o ejercido violencia de pareja. 


\section{REFERENCIAS BIBLIOGRÁFICAS}

ADIMARK (2014). El nivel socioeconómico ESOMAR. Manual de aplicación. Informe técnico, Chile, 2000 [ESOMAR socioeconomic status. Application manual. Technical report, Chile, 2000]. Recuperado el 22 de julio de 2014 de http://www.microweb.cl/idm/documentos/ESO MAR.pdf

Ard, K.L. \& Makadon, H.J. (2011). Addressing intimate partner violence in lesbian, gay, bisexual, and transgender patients. Journal of General Internal Medicine, 26(8), 930-933. http://dx. doi.org/10.1007/s11606-011-1697-6

Baker, N.L., Buick, J.D., Kim, S.R., Moniz, S. \& Nava, K.L. (2013). Lessons from examining same-sex intimate partner violence. Sex Roles, 69, 182-192. http://dx.doi.org/10.1007/s11199012-0218-3

Balsam, K.F., Rothblum, E.D. \& Beauchaine, T.P. (2005). Victimization over the life span: A comparison of lesbian, gay, bisexual, and heterosexual siblings. Journal of Consulting and Clinical Psychology, 73(3), 477-487.

Barrett, B. \& St. Pierre, M. (2013). Intimate partner violence reported by lesbian-, gay-, and bisexual-identified individuals living in Canada: An exploration of within-group variations. Journal of Gay \& Lesbian Social Services, 25 (1), 1-23. http://dx.doi.org/10.1080/10538720. 2013.751887

Barrientos, J. (2015). Violencia homofóbica en América Latina y Chile [Homophobic violence in Latin America and Chile]. Santiago de Chile: Ediciones y Publicaciones El Buen Aire S.A.

Barrientos, J. \& Bozón, M. (2014). Discrimination and victimization against gay men and lesbians in Chile: Two patterns or just one? Interdisciplinaria, 31(2), 323-339. http://dx.doi.org/10.1 6888/interd.2014.31.2.8

Barrientos, J. \& Cárdenas, M. (2014). Construction and validation of a subjective scale of stigma and discrimination (SISD) for the gay men and transgender women population in Chile. Sexuality Research \& Social Policy, 11(3), 187-
198. http://dx.doi.org/10.1007/s13178-014-01 50-0

Barrientos, J., Cárdenas, M. \& Gómez, F. (2014). Características sociodemográficas, bienestar subjetivo y homofobia en una muestra de hombres gay en tres ciudades chilenas [Sociodemographic characteristics, subjective well-being, and homophobia experienced by a sample of gay men from three cities in Chile]. Cadernos de Saúde Pública, 30(6), 1259-1269. http:// dx.doi.org/10.1590/0102-311X00108413

Barrientos, J., Meza, P., Gómez, F., Catalán, S., Longueira, J. \& Silva, J. (2008). Política, derechos, violencia y diversidad sexual: Primera encuesta Marcha por el Orgullo y Diversidad Sexual [Political, rights, violence and sexual diversity: First survey March for Pride and Sexual Diversity]. Santiago, Chile: CLAM/IM S/UERJ.

Barrientos, J., Rodríguez-Caballería, A., Escartín, J. \& Longares, L. (en prensa). Violencia en parejas del mismo sexo: Revisión y perspectivas actuales [Violence in same-sex couples: Review and outlook]. Revista Argentina de Clínica Psicológica.

Barrientos, J., Silva, J., Catalán, S., Gómez, F. \& Longueira, J. (2010). Discrimination and victimization: Parade for lesbian, gay, bisexual, and transgender (LGBT) pride, in Chile. Journal of Homosexuality, 57(6), 760-775. http:// dx.doi.org/10.1080/00918369.2010.485880

Burke, L.K. \& Follingstad, D.R. (1999). Violence in lesbian and gay relationships: Theory, prevalence, and correlational factors. Clinical Psychology Review, 19, 487-512. http://dx. doi.org/ 10.1016/S0272-7358(98)00054-3

Burke, T.W., Jordan, M.L. \& Owen, S.S. (2002). A cross-national comparison of gay and lesbian domestic violence. Journal of Contemporary Criminal Justice, 18(3), 231-257. http://dx.doi. org/10.1177/1043986202018003003

Cannon, C. \& Buttell, F. (2015). Illusion of inclusion: The failure of the gender paradigm to account for intimate partner. Partner Abuse, 6 (1), 65-77. http://dx.doi.org/10.1891/1946-65 60.6.1.65 
Coker, A.L., Smith, P.H., McKeown, R.E. \& King, M.J. (2000). Frequency and correlates of intimate partner violence by type: Physical, sexual, and psychological battering. American Journal of Public Health, 90, 553. http://dx. doi.org/10.2105/AJPH.90.4.553

Edwards, K.M. \& Sylaska, K.M. (2013). The perpetration of intimate partner violence among LGBTQ college youth: The role of minority stress. Journal of Youth and Adolescence, 42 (11), 1721-1731. http://dx.doi.org/10.1007/s10 964-012-9880-6

Ferreira, A.C., Simões, D., do Carmo, N. \& Moura, L. (2015). Violência entre parceiros íntimos: Uma comparação dos índices em relacionamentos hetero e homossexuais [Intimate partner violence: A comparison of indexes in relationships straight and gay]. Temas Em Psicologia, 23, 565-575. http://dx.doi.org/10.9788/ TP2015.3-04

Finneran, C., Chard, A., Sineath, C., Sullivan, P. \& Stephenson, R. (2012). Intimate partner violence and social pressure among gay men in six countries. The Western Journal of Emergency Medicine, 13(3), 260-271. http://dx.doi.org//10. 5811/westjem.2012.3.11779

Finneran, C. \& Stephenson, R. (2013). Intimate partner violence among men who have sex with men: A systematic review. Trauma, Violence, and Abuse, 14(2), 168-185. http://dx.doi.org/ $10.1177 / 1524838012470034$

Finneran, C. \& Stephenson, R. (2014). Intimate partner violence, minority stress, and sexual risk-taking among U.S. men who have sex with men. Journal of Homosexuality, 61, 288-306. http://dx.doi.org/10.1080/00918369.2013.8399 11

Garcia-Moreno, C., Jansen, H.A., Ellsberg, M., Heise, L., Watts, C.H. \& Study, W.H.O.M. (2006). Prevalence of intimate partner violence: findings from the WHO multi-country study on women's health and domestic violence. The Lancet, 368, 1260-69. http://dx.doi.org/10.101 6/ S0140-6736(06)69523-8

Guzmán, M., Espinoza, R., Tay, K., Leiva, J. \& Adaos, R. (2014). Evaluation of the mutuality of dating violence among university students from Antofagasta, Chile. En A. García (Ed.), Love, family and frienships: A Latin American perspective (pp. 63-77). Newcastle UK: Cambridge Scholars Publishing.

Harvey, A., Garcia-Moreno, C. \& Butchart, A. (2007). Primary prevention of intimate-partner violence and sexual violence: Background paper for WHO expert meeting. World Health Organization. Genova.

Hellemans, S., Loeys, T., Buysse, A., Dewaele, A. $\&$ de Smet, O. (2015). Intimate partner violence victimization among non-heterosexuals: Prevalence and associations with mental and sexual well-being. Journal of Family Violence, 30(2), 171-188. http://dx.doi.org/10.1007/s10896-01 5-9669-y

Letellier, P. (1994). Gay and bisexual male domestic violence victimization: Challenges to feminist theory and responses to violence. Violence and Victims, 9(2), 95-106.

Matte, M. \& Lafontaine, M.F. (2011). Validation of a measure of psychological aggression in same-sex couples: Descriptive data on perpetration and victimization and their association with physical violence. Journal of GLBT Family Studies, 7, 226-244. http://dx.doi.org/10.1 080/1550428X.2011.564944.

McClennen, J.C. (2005). Domestic violence between same-gender partners: Recent findings and future research. Journal of Interpersonal Violence, 20(2), 149-154. http://dx.doi.org/10. $1177 / 0886260504268762$

Messinger, A.M. (2011). Invisible victims: Samesex IPV in the National Violence Against Women Survey. Journal of Interpersonal Violence, 26(11), 2228-2243. http://dx.doi.org/10. $1177 / 0886260510383023$

Morrow, J. (1994). Identifying and treating battered lesbians. San Francisco Medicine, 17, 20-21.

ONUSIDA, Organización de las Naciones Unidas para la Educación, la Ciencia y la Cultura, Chile, Ministerio de Salud, VIVOPOSITIVO y ASOSIDA (2012). Índice compuesto de estigma y discriminación hacia hombres homosexuales, otros HSH y mujeres transgénero en 
Chile (ICED): Sintesis de estudio [Composite index of stigma and discrimination against gay men, other MSM and transgender women in Chile (ICED): Synthesis of study]. Santiago, Chile: Autores.

Owen, S.S. \& Burke, T.W. (2004). An exploration of prevalence of domestic violence in same-sex relationships. Psychological Reports, 95(1), 129-132. http://dx.doi.org/10.2466/PR0.95.5.1 29-132

Paz-Bailey, G., Miller, W., Shiraishi, R.W., Jacobson, J.O., Abimbola, T.O. \& Chen, S.Y. (2013). Reaching men who have sex with men: A comparison of respondent-driven sampling and time-location sampling in Guatemala City. AIDS and Behavior, 17(9), 3081-90. http://dx. doi. org/10.1007/s10461-013-0589-7

Poorman, P.B., Seelau, E.P. \& Seelau, S.M. (2003). Perceptions of domestic abuse in same-sex relationships and implications for criminal justice and mental health responses. Violence and Victims, 18, 659-669. http://dx.doi.org/10. 1891/vivi.2003.18.6.659

Ristock, J.L. (2003). Exploring dynamics of abusive lesbian relationships: Preliminary analysis of a multisite, qualitative Study1. American Journal of Community Psychology, 31, 329-341. http://dx.doi.org/10.1023/A:10239710068 82.

Russell, B. (2015). Bridging the gap in lnowledge about partner abuse in LGBTQ populations. Partner Abuse, 6(1), 3-7. http://dx.doi.org/10.1 891/1946-6560.6.1.3

Schechory, M. \& Ziv, R. (2007). Relationships between gender role attitudes, role division, and perception of equity among heterosexual, gay and lesbian couples. Sex Roles, 56, 629-638. http://dx.doi.org/10.1007/s11199-007-9207-3

Turell, S.C. (2000). A descriptive analysis of samesex relationship violence for a diverse sample. Journal of Family Violence, 15(3), 281-293. http://dx.doi.org/10.1023/A:1007505619577

Escuela de Psicología, Universidad Católica del Norte, Antofagasta Escuela de Psicología, Universidad de Santiago de Chile, Santiago Escuela de Psicología, Universidad de Valparaíso, Valparaiso Chile 